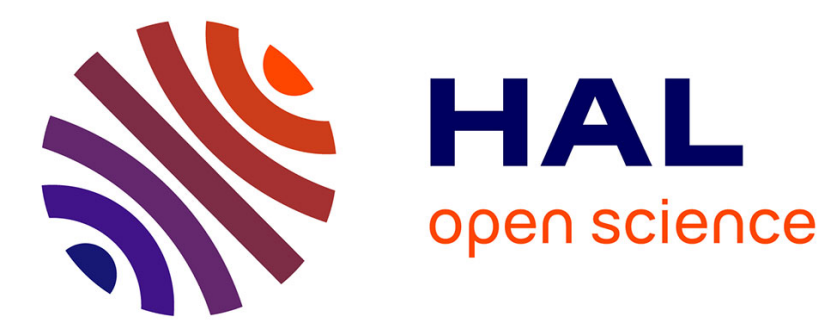

\title{
Processus catalytiques dans les réacteurs à plasma hors d'équilibre. III. Décomposition de NH3
}

\author{
D. Rapakoulias, J. Amouroux, M. P. Bergougnan, A. Gicquel
}

\section{To cite this version:}

D. Rapakoulias, J. Amouroux, M. P. Bergougnan, A. Gicquel. Processus catalytiques dans les réacteurs à plasma hors d'équilibre. III. Décomposition de NH3. Revue de Physique Appliquée, 1982, 17 (2), pp.95-101. 10.1051/rphysap:0198200170209500 . jpa-00244977

\section{HAL Id: jpa-00244977 https://hal.science/jpa-00244977}

Submitted on 1 Jan 1982

HAL is a multi-disciplinary open access archive for the deposit and dissemination of scientific research documents, whether they are published or not. The documents may come from teaching and research institutions in France or abroad, or from public or private research centers.
L'archive ouverte pluridisciplinaire HAL, est destinée au dépôt et à la diffusion de documents scientifiques de niveau recherche, publiés ou non, émanant des établissements d'enseignement et de recherche français ou étrangers, des laboratoires publics ou privés. 


\title{
Processus catalytiques dans les réacteurs à plasma hors d'équilibre III. Décomposition de $\mathrm{NH}_{3}$
}

\author{
D. Rapakoulias, J. Amouroux, M. P. Bergougnan et A. Gicquel \\ ENSCP, Laboratoire de Génie Chimique, 11, rue Pierre-et-Marie-Curie, 75231 Paris Cedex 05, France
}

(Reçu le 24 juillet 1981, révisé le 14 octobre, accepté le 20 octobre 1981)

\begin{abstract}
Résumé. - Nous avons tout d'abord établi l'existence de phénomènes catalytiques dus à l'introduction d'une plaque métallique $(\mathrm{W}, \mathrm{Mo})$ dans un plasma à pression réduite.

Nous avons ensuite mis en évidence l'existence d'une couche limite, au voisinage du catalyseur, dans laquelle la répartition énergétique est différente de celle du gros du plasma.

La comparaison de ces résultats expérimentaux avec les calculs théoriques effectués par Wolken, a permis de dégager le rôle des différentes étapes de la chimisorption.

Lors de la recombinaison-désorption il y a apparition d'une énergie excédentaire qui peut se répartir entre le gaz et le solide.

Ce transfert d'énergie est la cause d'un mécanisme rétroactif de la chimisorption et de l'échauffement du métal.
\end{abstract}

\begin{abstract}
First we have established the existence of catalytic phenomena due to the introduction of a metallic foil (W, Mo) in a reduced pressure plasma.

Then we have shown the existence of a limit layer, nearest to the catalyst, within the energy dispersal is different from the bulk of the plasma.

The comparison of the experimental results with the theoretical calculations made by Wolken, led to the role of the different steps of the chemisorption.

During the recombination-desorption, there is an excess energy which appears, and which can be divided between the gas and the solid.

This energy transfer is the cause of a feed-back mechanism of the chemisorption, and of the heating of the solid.
\end{abstract}

1. Introduction. - La présence d'une surface métallique dans un réacteur à plasma peut donner lieu à des réactions de surface catalytiques. Les réactions de ce type étudiées expérimentalement comprennent la synthèse de $\mathrm{HCN}[1]$, de $\mathrm{NO}[2,13]$, de $\mathrm{NH}_{3}$ [6], d'anhiline [11], d'hydrazine [12] et la décomposition d'hydrocarbures [14]. D'autres cas particuliers de catalyse dans les décharges électriques apparaissent lors du traitement des films polymériques et la production d'ozone $[15,16]$.

Les plasmas à pression réduite sont des milieux hors d'équilibre. La réactivité chimique de,ces milieux est différente de celle des systèmes à l'équilibre thermodynamique. Elle dépend non seulement de la quantité d'énergie disponible, mais aussi de sa répartition sur les différents modes d'excitation des molécules $[1,17]$.

Les règles de base régissant la réactivité chimique en milieu hors d'équilibre en phase gazeuse ont été établies par Polanyi [17] dans le cas de systèmes simples, où seulement deux modes d'excitation interne (vibration et rotation) sont activés. Nous avons cherché à étendre ces règles aux réactions dans les plasmas basse pression, qui sont des milieux plus complexes [1, 4]. Notre conclusion est que l'extension aux plasmas des règles de Polanyi est possible sous deux conditions : premièrement, de tenir compte aussi de l'excitation électronique des molécules, dont la grande réactivité provient de leur symétrie spatiale différente de celle du fondamental. Deuxièmement de ne pas raisonner sur la réaction globale, mais de considérer séparément les trois étapes de la réaction (excitation, réacteur chaud, trempe).

Lorsque la réaction a lieu sur la surface d'un catalyseur il convient de penser que l'efficacité du phénomène catalytique pourra être modifiée selon le type de l'excitation (vibration ou translation) des réactifs. 
Wolken $[7,8]$ a été le seul à étudier l'influence de l'excitation hors d'équilibre sur les réactions catalytiques hétérogènes. Il s'agit de travaux théoriques établissant l'influence de l'excitation vibrationnelle et translationnelle, selon la forme de la surface d'énergie potentielle du système gaz-solide.

Nous allons dans cet article confirmer l'étude expérimentale de la relation entre l'effet catalytique et l'excitation interne du plasma, commencée par l'étude de la synthèse de NO [2] et de HCN [4]. Nous pourrons ainsi conclure dans quelle mesure les hypothèses de Wolken peuvent être appliquées aux réactions catalytiques hétérogènes en phase plasma.

Le système expérimental choisi est la décomposition de $\mathrm{NH}_{3}$ sur le W et le Mo. En effet, outre son intérêt pratique (production de $\mathrm{H}_{2}$ ultra-pur, régénération de catalyseurs), cette réaction endothermique nous permet d'étudier les processus catalytiques, de formation de $\mathrm{N}_{2}$ et de les comparer aux études précédentes sur la fixation de $\mathrm{N}_{2}$.

Nous allons tout d'abord établir expérimentalement l'existence de phénomènes catalytiques dus à la présence de métaux introduits dans le plasma. Nous proposerons ensuite un mécanisme de catalyse à partir de l'étude parallèle de la réactivité chimique et de l'analyse spectroscopique du plasma en présence de catalyseur.

Enfin nous comparerons nos conclusions sur la relation entre l'excitation interne des molécules dans le plasma et l'effet catalytique avec les calculs théorisques effectués par Wolken. Nous apportons ainsi la première confirmation expérimentale de ces calculs, et à partir de cette conclusion et les travaux de Halpern et Rosner [9] nous envisageons l'aspect énergétique du processus de catalyse et du rôle de la nature du catalyseur.

2. Dispositif expérimental et mode opératoire. - Le dispositif expérimental est identique à celui utilisé pour l'étude de la décomposition de $\mathrm{NH}_{3}$ sans catalyse [3] : un plasma d'ammoniac est créé par une décharge $\mathrm{HF}$ $(40 \mathrm{MHz})$ à pression réduite (5 à $40 \mathrm{mbar}$ ).

Le réacteur proprement dit est illustré sur la figure 1. Il s'agit d'un cylindre de $0,1 \mathrm{~m}$ de diamètre délimité par la self inductrice et la paroi de trempe refroidie. Le débit d'alimentation varie entre 0,5 et $21 / \mathrm{min}$, ce qui correspond à un temps de passage compris entre 0,3 et 1,2 s. La puissance disponible aux bornes de l'inducteur est comprise entre 0,5 et $2,5 \mathrm{~kW}$. Le catalyseur est introduit dans la torche, à $2 \mathrm{~cm}$ de la paroi de trempe. Il est constitué d'une plaque métallique percée, et soutenu par 2 tiges en acier inoxydable fixées sur la paroi de trempe. Les métaux testés sont le molybdène et le tungstène.

Les gaz sortant sont analysés par chromatographie en phase vapeur [3]. Pour détecter les phénomènes catalytiques, chaque expérience est effectuée deux fois : une fois sans catalyseur, une fois en sa présence. Nous

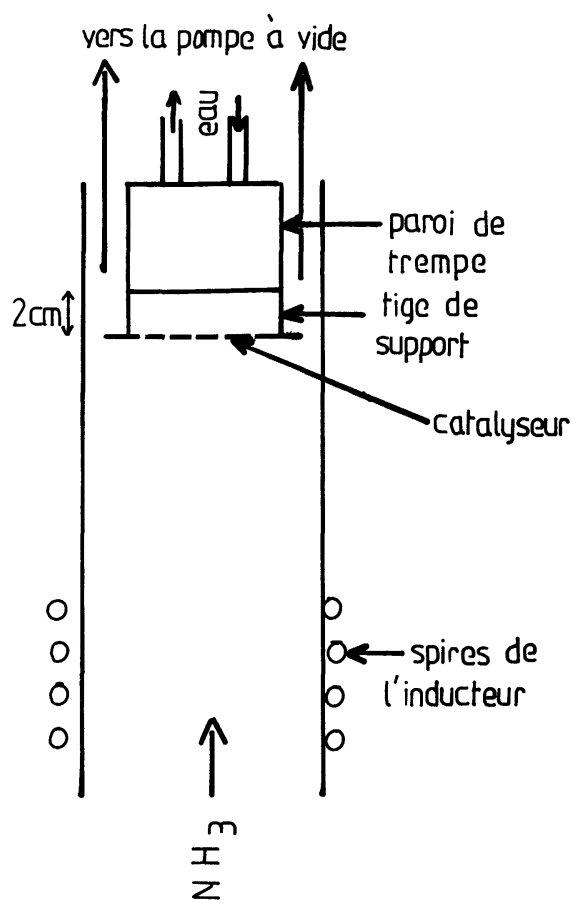

Fig. 1. - Schéma du réacteur catalytique.

[Schema of the catalytic reactor.]

comparons ensuite le taux de décomposition de $\mathrm{NH}_{3}$ défini par la relation :

$$
x=\frac{\left(\mathrm{NH}_{3}\right)^{0}-\left(\mathrm{NH}_{3}\right)}{\left(\mathrm{NH}_{3}\right)^{0}}
$$

Les mesures des températures de vibration et de rotation ont été effectuées par spectrométrie d'émission, sur un monochromateur JOBIN-YVON de $0,6 \mathrm{~m}$ de focale, muni d'un réseau de $2400 \mathrm{tr} / \mathrm{min}$.

Le plasma de $\mathrm{NH}_{3}$ est stable dans la région expérimentale étudiée. Sa couleur varie du blanc au jaune clair, dans le corps du plasma; et parallèlement à la paroi du catalyseur on observe une zone de l'ordre de $1 \mathrm{~cm}$ de couleur plus intense (couche limite).

La molécule de $\mathrm{NH}_{3}$ ne donne pas de spectre d'émission uv-visible dans les décharges. Parmi les produits de décomposition, les transitions observées les plus intenses sont le deuxième système positif de $\mathrm{N}_{2}$ et la transition $\mathrm{A}^{3} \Pi \rightarrow \mathrm{X}^{3} \Sigma^{-}$de $\mathrm{NH}$. Ces deux transitions permettent la mesure des températures de vibration et de rotation-translation, celles-ci étant supposées homogènes sur le diamètre du réacteur. La première est mesurée sur les bandes de transition $\Delta v=2$ du deuxième système positif de $\mathrm{N}_{2}$ et la seconde sur les raies de la bande $0-0$ de la transition $\mathrm{A}^{3} \Pi \rightarrow \mathrm{X}^{3} \Sigma$ de NH. Pour le calcul de ces températures nous avons utilisé la méthode graphique de Boltzmann.

On observe d'autres transitions intenses correspondant à celles déjà citées dans une étude précédente [3]. Nous rappellerons simplement que nous n'avons pas détecté les bandes Schuster caractéristiques de la formation d'hydrazine. 
3. Rappels des conclusions de l'étude du réacteur sans catalyse. - Dans une étude précédente (3), le système réactionnel sans catalyse a été étudié. Parmi les conclusions de cette étude, il nous sera utile d'en rappeler les suivantes :

a) La molécule d'ammoniac, excitée dans la décharge produit 2 catégories d'états excités : les états électroniquement excités dissociatifs $\left(\mathrm{NH}_{3}\right)^{* *}$ et l'état fondamental possédant une forte excitation sous forme d'énergie vibrationnelle $\left(\mathrm{NH}_{3}\right)^{*}$ mais ne participant à aucune réaction.

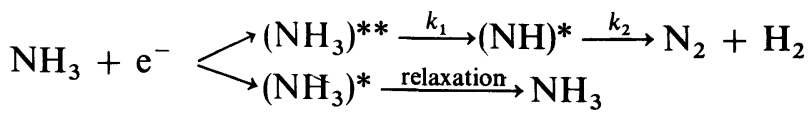

b) La voie de décomposition: $\mathrm{NH}_{3} \rightarrow \mathrm{NH}_{2}+\mathrm{H}$, n'a pas été retenue.

c) La constante de vitesse $k_{1}$ est très inférieure à $k_{2}$.

d) L'étape déterminante de la décomposition de $\mathrm{NH}_{3}$ est l'étape endothermique $\left(\mathrm{NH}_{3}\right)^{* *} \rightarrow(\mathrm{NH})^{*}$, contrôlée par la température de vibration.

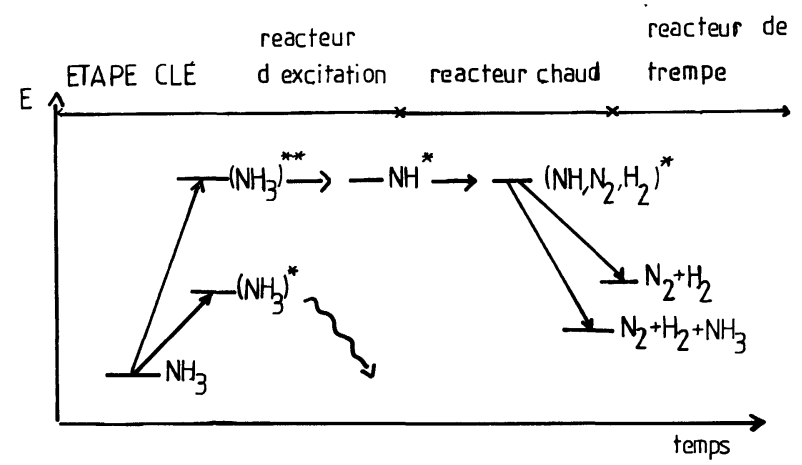

Fig. 2. - Diagramme énergétique pour la décomposition de $\mathrm{NH}_{3}$ en phase gazeuse.

[Energy diagram. Gas phase.]

Les conclusions sur le réacteur sans catalyse ont permis de proposer le schéma de la figure 2 .

4. Mise en évidence du phénomène catalytique. Les figures 3,4 et 5 représentent le taux de décomposi-

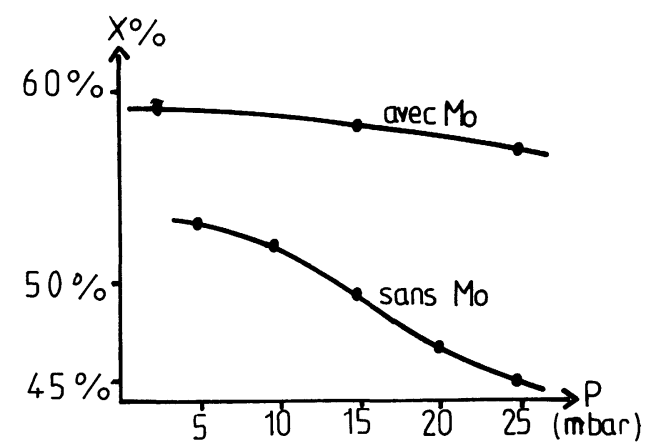

Fig. 3. - Variation du taux de décomposition de $\mathrm{NH}_{3}$ en fonction de la pression, en présence ou en absence d'une. plaque métallique (Mo) $\left(D_{\mathrm{NH}_{3}}=0,5 \mathrm{l} / \mathrm{min}, P I=0,8 \mathrm{~kW}\right)$. [Variation of the $\mathrm{NH}_{3}$ decomposition rate with pressure, in presence or in absence of a metallic foil (Mo) $\left(D_{\mathrm{NH}_{3}}=0,51 / \mathrm{min} ., P I=0.8 \mathrm{~kW}\right)$.]

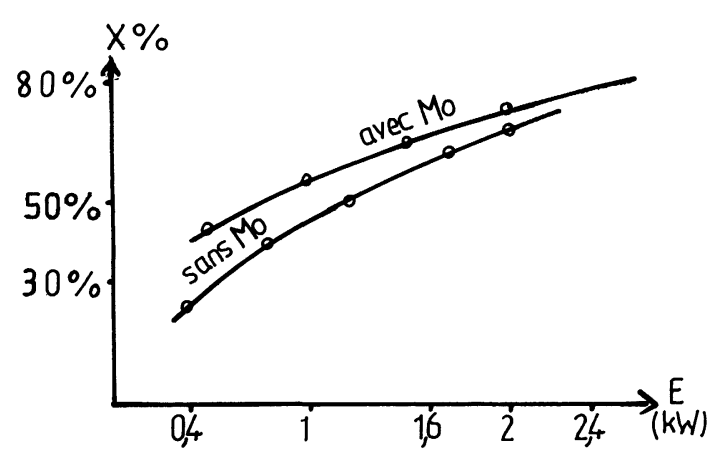

Fig. 4. - Variation du taux de décomposition de $\mathrm{NH}_{3}$ en fonction de la puissance induite, en présence ou en absence d'une plaque métallique (Mo) $\left(P=5 \mathrm{mbar}, D_{\mathrm{NH}_{3}}=0,5 \mathrm{l} / \mathrm{min}\right)$.

[Variation of the $\mathrm{NH}_{3}$ decomposition rate with induced power, in presence or in absence of a metallic foil (Mo) $\left(P=5 \mathrm{mbar}, D_{\mathrm{NH}_{3}}=0.5 \mathrm{l} / \mathrm{min}\right.$.). $]$

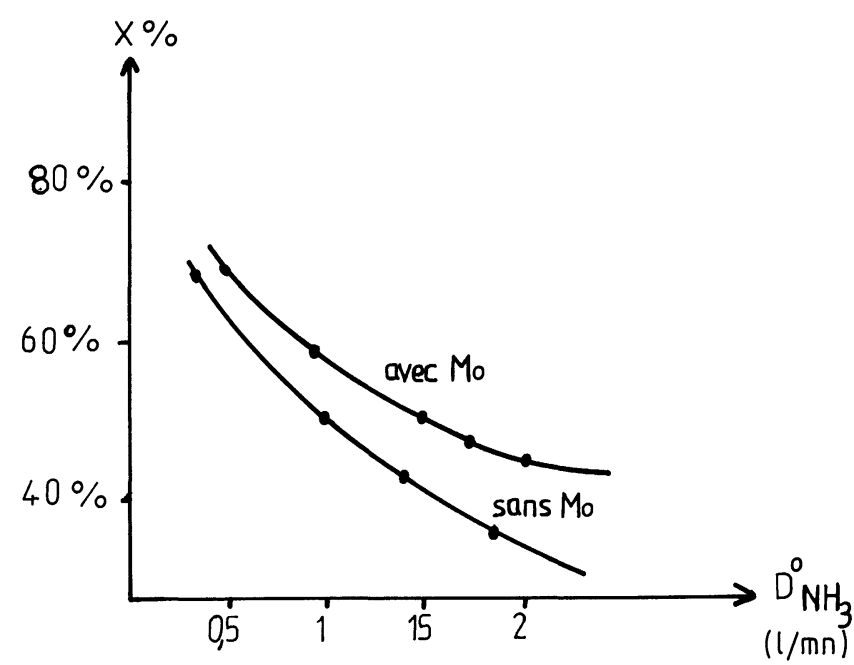

Fig. 5. - Variation du taux de décomposition de $\mathrm{NH}_{3}$ en fonction du débit de $\mathrm{NH}_{3}$ à l'entrée du réacteur, en présence ou en absence d'une plaque métallique $(\mathrm{Mo})(P=5 \mathrm{mbar}$, $P I=0,8 \mathrm{~kW}$ ).

[Variation of the $\mathrm{NH}_{3}$ decomposition rate with the $\mathrm{NH}_{3}$ flow introduced in presence or in absence of a metallic foil (Mo) $(P=5 \mathrm{mbar}, P I=0.8 \mathrm{~kW})$.]

tion de $\mathrm{NH}_{3}$ en présence ou en absence d'une plaque mécanique Mo.

Ces résultats font apparaître une augmentation de la décomposition de $\mathrm{NH}_{3}$ en présence d'une plaque métallique. Il s'agit donc d'un phénomène catalytique tel qu'il a été défini dans un réacteur à plasma.

5. Interprétation du phénomène catalytique dans le réacteur. - 5.1 RECHERCHE D'UN SCHÉMA DE CATALYSE. - Le rendement total d'une réaction en phase plasma peut être amélioré par 4 voies différentes déjà décrites dans une étude précédente [4].

a) Augmentation de la population de $\left(\mathrm{NH}_{3}\right)^{* *}$ par transfert sélectif au moyen d'un donneur.

b) Augmentation de la durée de vie de $\left(\mathrm{NH}_{3}\right)^{* *}$ par absorption sur une surface. 
c) Stabilisation du produit de la réaction $(\mathrm{NH}$, $\left.\mathrm{N}_{2}, \mathrm{H}_{2}\right)^{*}$ par désexcitation sur une surface.

d) Activation de l'état de faible énergie $\mathrm{NH}_{3}^{*}$ par chimisorption sur une surface.

C'est cette dernière voie qui est responsable de la catalyse dans notre réacteur. En effet, la voie $a$ ) est exclue car il n'y a pas de donneur pour un transfert sélectif, et les deux autres voies peuvent être éliminées comme nous allons le démontrer.

5.2 Confirmation eXpérimentale. - Dans le réacteur sans catalyse, comme dans celui avec catalyse, le rendement de la décomposition est amélioré

eau

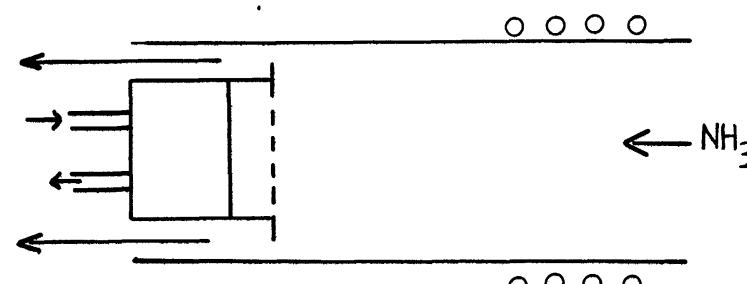

$\underset{\text { région étudiée }}{ }$
par spectrométrie
d’emission

Fig. 6. - Délimitation de la région étudiée par spectrométrie d'émission.

[Delimitation of the studied aera by emission spectrometry.] par une faible pression (Fig. 3), c'est-à-dire une forte valeur de la température de vibration car ces 2 facteurs varient en sens inverse [3].

L'étude de la température de vibration dans le réacteur permet d'étudier le mécanisme de la catalyse. La température de vibration a été mesurée dans une large zone du réacteur comme l'indique la figure 6.

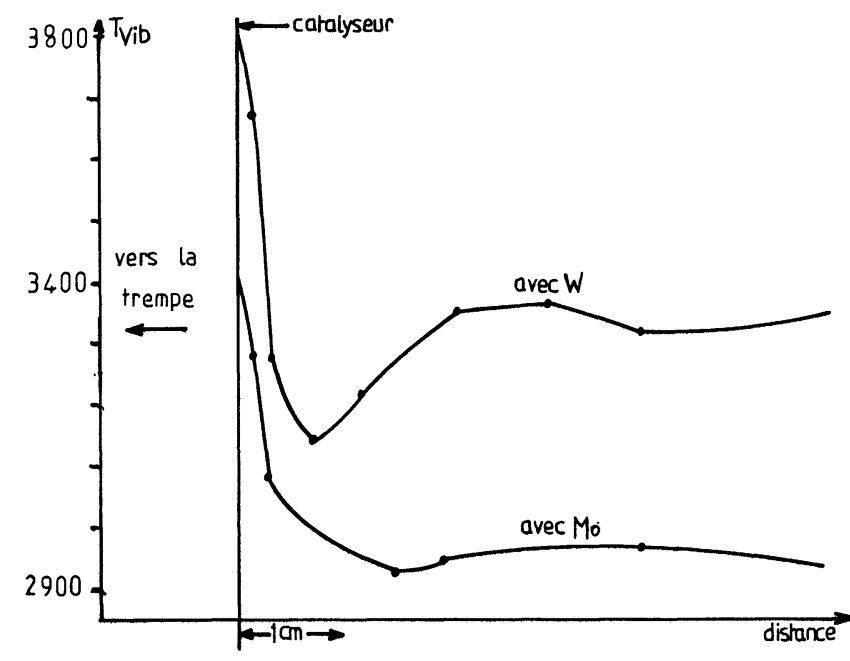

Fig. 7a. - Variation de la température de vibration dans le réacteur, en présence de la plaque métallique (W ou Mo) $\left(P=5 \mathrm{mbar}, P I=0,8 \mathrm{~kW}, D_{\mathrm{NH}_{3}}=0,51 / \mathrm{min}\right)$.

[Variation of the vibrational temperature in the reactor, in presence of a metallic foil (W or Mo) $(P=5$ mbar, $P I=0.8 \mathrm{~kW}, D_{\mathrm{NH}_{3}}=0.51 / \mathrm{min}$.).]

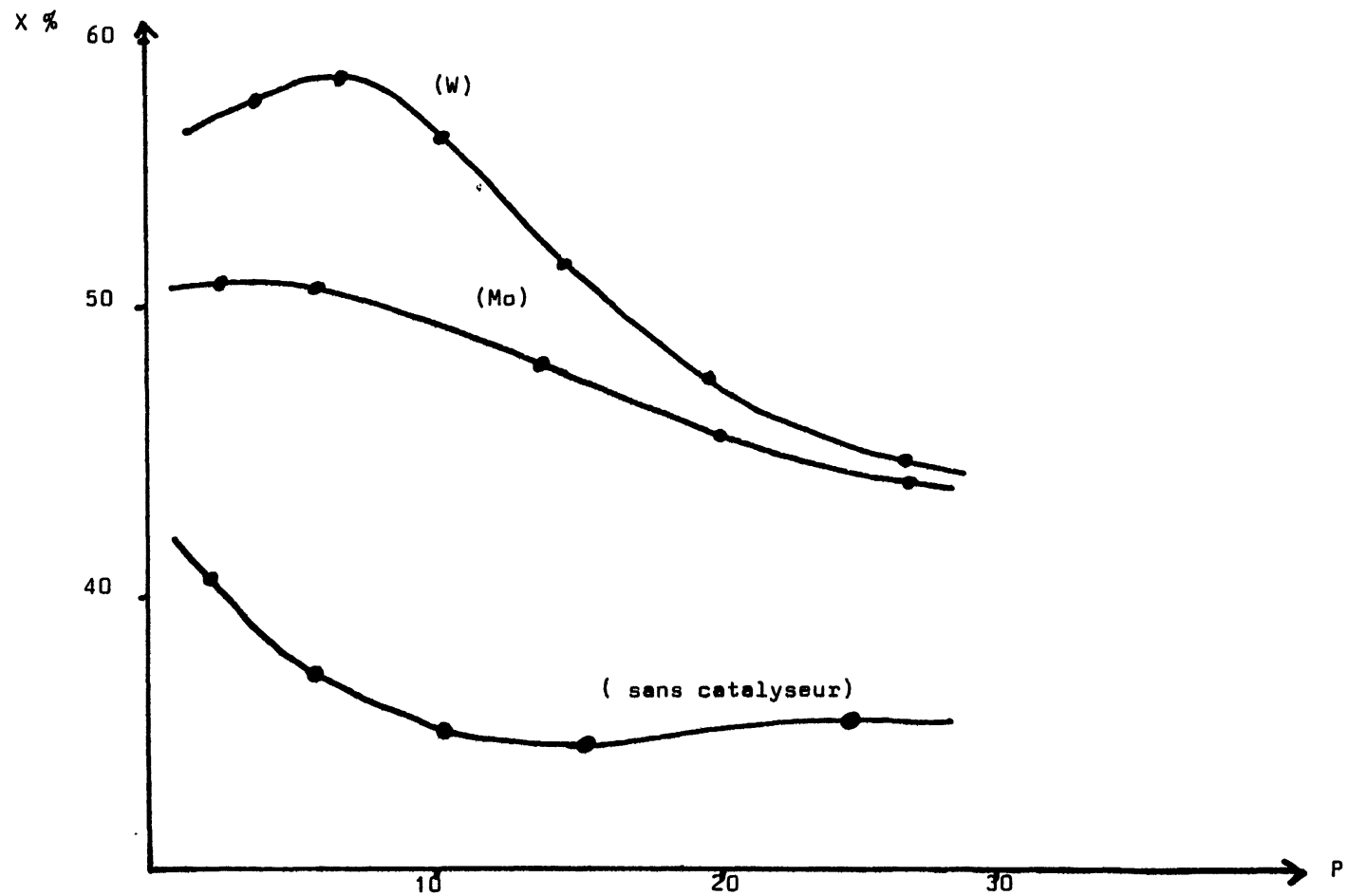

Fig. 7b. - Variation du taux de décomposition de $\mathrm{NH}_{3}$ en fonction de la pression (mbar) selon la nature du catalyseur $(\mathrm{W}$ ou $\mathrm{Mo})\left(P I=0,98 \mathrm{~kW}, D_{\mathrm{NH}_{3}}^{0}=11 / \mathrm{min}\right)$.

[Variation of the $\mathrm{NH}_{3}$ decomposition rate with respect to the pressure (mbars) according to the catalyst nature (W or Mo) $\left(P I=0.98 \mathrm{~kW}, D_{\mathrm{NH}_{3}}^{0}=1 \mathrm{l} / \mathrm{min}.\right)$. $]$ 
Ces mesures ont été effectuées en présence de catalyseur (plaque métallique de Mo et de W); figure 7.

Ce résultat fait apparaître qu'il s'agit bien d'un phénomène de chimisorption; en effet la température de vibration dépend du métal utilisé comme catalyseur. Par conséquent les voies $b$ ) et $c$ ) sont à éliminer. En effet l'augmentation de la durée de vie de $\left(\mathrm{NH}_{3}\right)^{* *}$ par absorption sur une surface ou la désexcitation du produit de la réaction $\left(\mathrm{NH}, \mathrm{N}_{2}, \mathrm{H}_{2}\right)^{*}$ sur une surface ne nécessitent pas la création de liaisons fortes (chimisorption) entre le métal et la molécule gazeuse mais seulement un contact physique. De ce fait l'effet catalytique ne devrait pas dépendre de la nature du métal; or les résultats expérimentaux indiquent une influence marquée de la nature du métal (Fig. $7 a$ et $7 b$ ).

Il ne reste donc comme voie possible pour la catalyse que l'activation de l'ammoniac par un processus de chimisorption à partir des états de faible énergie (voie $d)$ ). Ce mécanisme est confirmé par un autre résultat expérimental. En effet il implique une baisse du taux de $\mathrm{NH}$ par rapport au taux de $\mathrm{N}_{2}$ au niveau $\mathrm{du}$ catalyseur. Cette diminution du rapport $\mathrm{NH} / \mathrm{N}_{2}$ a été confirmée expérimentalement (Fig. 8). Le rapport

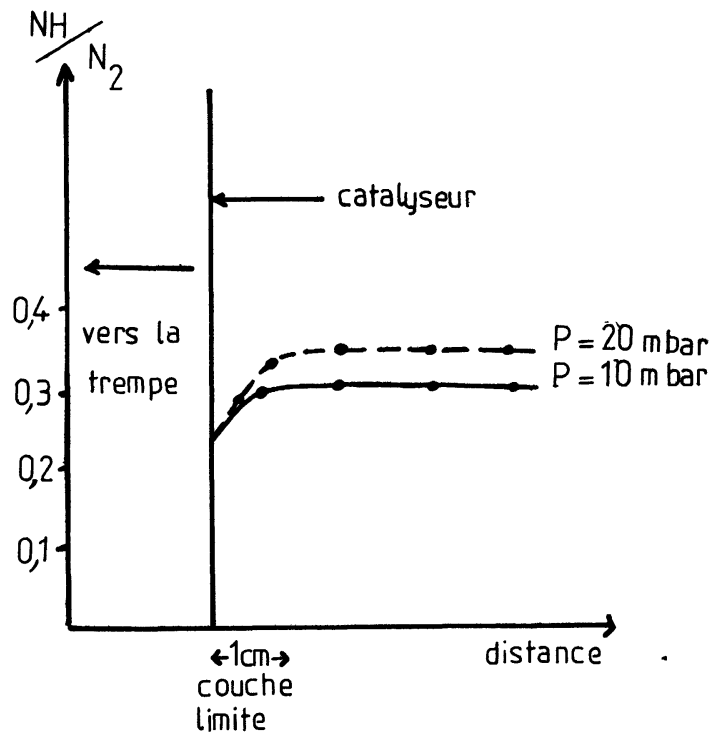

Fig. 8. - Variation du rapport $\mathrm{NH} / \mathrm{N}_{2}$ en présence d'une plaque métallique (W) $(P=5$ mbar, $P I=0,8 \mathrm{~kW}$, $D_{\mathrm{NH}_{3}}=0,5 \mathrm{l} / \mathrm{min}$ ).

[Variation of the $\mathrm{NH} / \mathrm{N}_{2}$ rate in presence of a metallic foil (W) $\left(P=5 \mathrm{mbar}, P I=0.8 \mathrm{~kW}, D_{\mathrm{NH}_{3}}=0,5 \mathrm{l} / \mathrm{min}\right.$.). $]$

$\mathrm{NH} / \mathrm{N}_{2}$ a été évalué d'après le rapport des têtes de bandes de la transition $\mathrm{C}^{3} \Pi_{\mathrm{u}} \rightarrow \mathrm{B}^{3} \Pi_{\mathrm{g}}$ (bande 0-0) de $\mathrm{N}_{2}$ et de la transition $\mathrm{A}^{3} \Pi \rightarrow \mathrm{X}^{3} \Sigma^{-}$(bande 0-0) de NH. Tous ces résultats nous permettent de proposer le diagramme énergétique de la figure 9.

L'étude du système sans catalyse a démontré que la décomposition de $\mathrm{NH}_{3}$ augmente avec l'écart à l'équilibre $\alpha$ (paramètre défini par la relation $\alpha=T_{\mathrm{v}}-T_{\mathrm{r}} / T_{\mathrm{v}}+T_{\mathrm{r}}$ ) c'est-à-dire lorsque la tem-

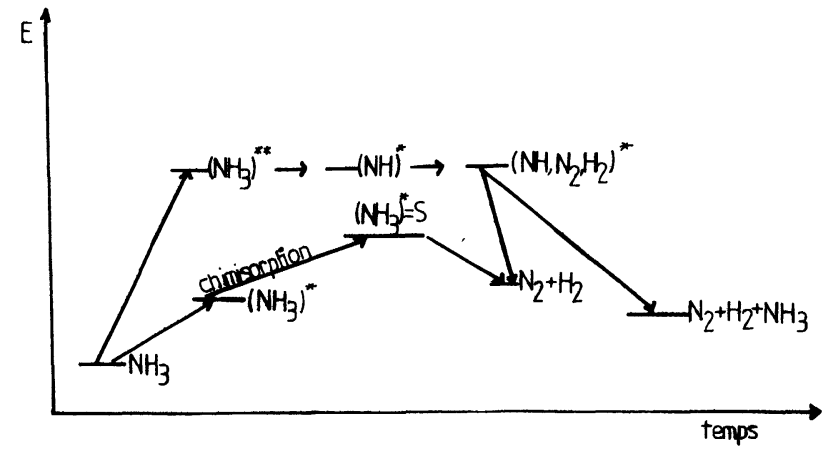

Fig. 9. - Diagramme énergétique pour la décomposition de $\mathrm{NH}_{3}$ en phase catalytique.

[Energy diagram. Catalytic phase.]

pérature de vibration s'élève (pression faible). On observe le même phénomène dans le réacteur catalytique (Fig. 10). Mais pour l'efficacité de la catalyse (Fig. 10), cette relation est inversée. En effet, à forte température de vibration, l'étape-clé de la réaction en phase gazeuse et la réaction de surface sont toutes deux favorisées; or la réaction en phase gazeuse étant

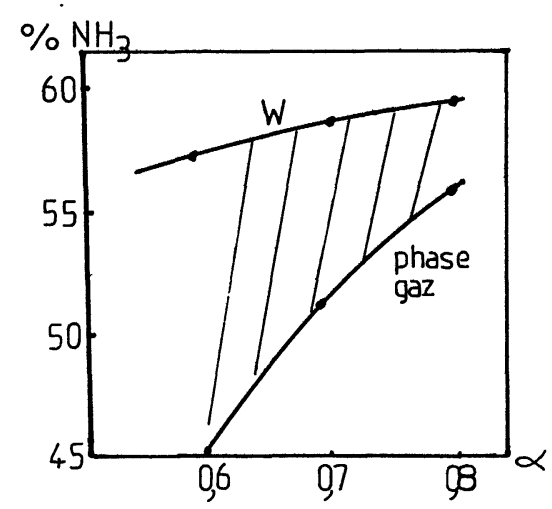

Fig. 10. - Variation du taux de décomposition de $\mathrm{NH}_{3}$ en fonction de l'écart à l'équilibre $\alpha$, en présence ou en absence d'une plaque métallique (W)

$$
\alpha=\frac{T_{\mathrm{vib}}-T_{\text {trans }}}{T_{\mathrm{M}}} \quad T_{\mathrm{M}}=T_{\mathrm{vib}}+T_{\text {trans }} .
$$

Partie hachurée : efficacité catalytique.

[Variation of the $\mathrm{NH}_{3}$ decomposition rate with the equilibrium divergence $\alpha$, in presence or in absence of a metallic foil (W)

$$
\alpha=\frac{T_{\mathrm{vib}}-T_{\text {trans }}}{T_{\mathrm{M}}} \quad T_{\mathrm{M}}=T_{\mathrm{vib}}+T_{\text {trans }} .
$$

Dashed area : catalytic efficiency.]

plus rapide que la réaction de surface, elle l'emporte sur la voie catalytique et par conséquent l'effet catalytique est moins fort à température de vibration élevée qu'à faible température de vibration, où la réaction prédominante est la réaction de surface. 
6. Rôle de l'excitation vibrationnelle dans la catalyse. - 6.1 Résultats EXPéRIMENTAUX. - Pour conclure ce travail nous avons cherché à établir les relations entre la nature hors d'équilibre du plasma et l'effet catalytique.

Pour cela nous avons comparé nos résultats expérimentaux aux calculs théoriques effectués par Wolken $[7,8]$ sur la dynamique d'adsorption et de désorption en milieu hors d'équilibre.

Les principales conclusions des travaux de Wolken, basés sur un modèle mathématique où les paramètres énergies vibrationnelle, rotationnelle et translationnelle ne sont liés que par la contrainte de la conservation de l'énergie totale, et où par conséquent énergies rotationnelle et translationnelle peuvent prendre des valeurs différentes, sont les suivantes : l'adsorption est en général favorisée par une forte température de vibration et les produits désorbés sont fortement vibrationnellement excités. Lors de la désorption il y a une forte augmentation de la température de vibration et une faible augmentation de la température de rotation au détriment de la température de translation.

Expérimentalement, nous avons déjà démontré (Fig. 10) qu'effectivement le pourcentage de décomposition de $\mathrm{NH}_{3}$ augmente avec la température de vibration, c'est-à-dire lorsque le phénomène d'adsorption est favorisé puisque la catalyse est régie par un phénomène de chimisorption.

De même nous avons mis en évidence (Fig. 11) dans notre réacteur, au voisinage du catalyseur dans une zone de l'ordre de $1 \mathrm{~cm}$ appelée couche

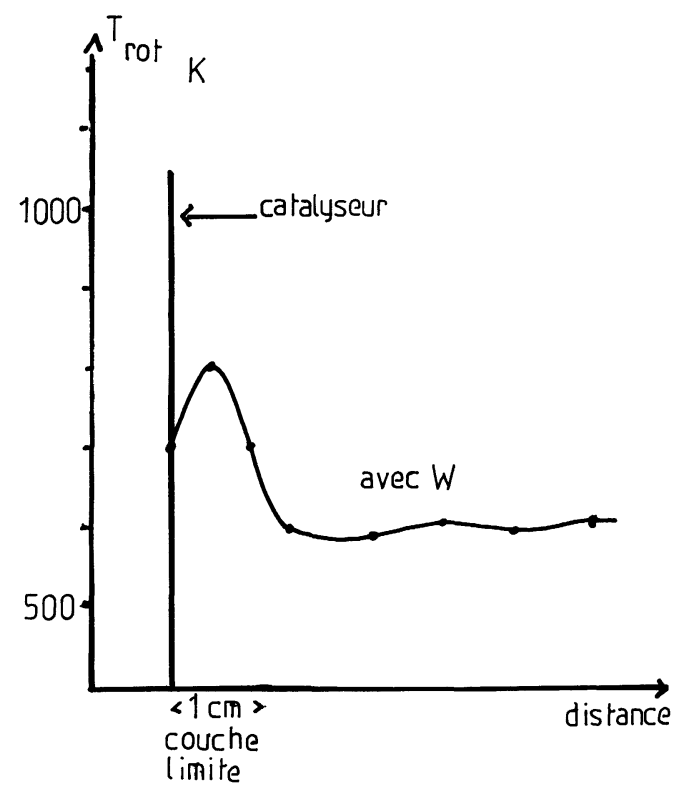

Fig. 11. - Variation de la température de rotation dans le réacteur, en présence d'une plaque métallique (W) $\left(P=5 \mathrm{mbar}, P I=0,8 \mathrm{~kW}, D_{\mathrm{NH}_{3}}=0,5 \mathrm{l} / \mathrm{min}\right)$.

[Variation of the rotational temperature in the reactor, in presence of a metallic foil (W) $(P=5 \mathrm{mbar}, P I=0.8 \mathrm{~kW}$, $D_{\mathrm{NH}_{3}}=0.5 \mathrm{l} / \mathrm{min}$.).] limite, une forte augmentation de l'excitation vibrationnelle (Fig. 7) et aussi une augmentation plus faible de l'excitation rotationnelle (Fig. 11).

Ces résultats, qui représentent la première confirmation expérimentale de calculs théoriques effectués sur les phénomènes d'adsorption et de désorption, mettent en évidence l'existence d'une relation étroite entre l'effet catalytique et la nature hors d'équilibre du plasma. A la différence d'une catalyse en milieu thermique, dans un milieu hors d'équilibre le catalyseur modifie la répartition énergétique à son voisinage, créant ainsi une couche limite où sont exploités les états de faible énergie.

6.2 ASPECT ÉNERGÉTIQUE. - A partir de ces résultats, on peut envisager les deux principaux aspects du catalyseur.

Tout d'abord, il se comporte comme une surface où a lieu le phénomène de chimisorption.

Ensuite il conserve une part de l'excès d'énergie ce qui stabilise la molécule désorbée.

Considérons en premier la chimisorption : elle est activée par un mécanisme de transfert d'excitation rétroactif dans la couche limite.

En effet la couche limite possède une forte excitation vibrationnelle créé par les molécules d'azote désorbées dont l'excitation vibrationnelle est très élevée. Une partie de cette énergie vibrationnelle est transférée aux molécules de $\mathrm{NH}_{3}$ qui pénètrent dans la couche limite, accélérant de ce fait leur adsorption sur le métal. L'étape d'adsorption est donc accélérée grâce à des transferts sélectifs (vibration-vibration) dans la couche limite, à partir de l'énergie vibrationnelle libérée lors de la désorption.

Pendant la désorption des produits un excès d'énergie apparaîtra si l'énergie de la liaison créée dépasse l'enthalpie de désorption. Halpern et Rosner [9] ont étudié les conditions de partage de cet excès d'énergie entre la molécule désorbée et le solide. En effet lors de la recombinaison de 2 atomes d'azote, il y a création d'une liaison $\mathrm{N}-\mathrm{N}$ et rupture des liaisons $\mathrm{N}$-métal. Par exemple, dans le cas du tungstène, la rupture des 2 liaisons $\mathrm{N}-\mathrm{W}$ nécessite une énergie d'environ $8 \mathrm{eV}$ et l'énergie libérée par la création de la liaison $\mathrm{N}-\mathrm{N}$ est de $9,7 \mathrm{eV}$. L'énergie excédentaire $(1,7 \mathrm{eV})$ peut donc se répartir entre le métal et la molécule d'azote désorbée qui est alors vibrationnellement excitée.

La répartition de cette énergie excédentaire, entre le gaz et le solide est fonction de la nature du solide [9, 10], du gaz, du mécanisme réactionnel (LangmuirHinshelwood ou Rideal), et de la température de translation du plasma [9].

Wolken [10] qui a aussi abordé ce problème de transfert d'énergie, a prévu que la température de Debye et le transfert d'énergie du gaz vers le solide varient en sens opposé. Expérimentalement nous avons retrouvé l'influence de ce facteur sur le transfert d'énergie. En effet nous avons vu (Fig. 7) que la valeur de la température de vibration est beaucoup plus 
élevée dans le réacteur, en présence de W que de Mo. Cela est dû au fait que la température de Debye du tungstène est supérieure à celle du molybdène. Par conséquent, et en accord avec Wolken, le transfert d'énergie vers le solide est inférieur dans le cas du tungstène et lors de la désorption, la majeure partie de l'énergie excédentaire est acquise par la molécule gazeuse.

A ce transfert, s'y ajoute un second transfert de l'excitation vibrationnelle des molécules gazeuses de la couche limite vers le catalyseur. Ce type de transfert est en général peu efficace. Il est toutefois facilité lorsque des espèces physisorbées (en quasirésonance vibrationnelle avec les molécules de gaz) jouent le rôle d'intermédiaire. Dans notre réacteur, de telles espèces physisorbées peuvent correspondre aux états précurseurs de la chimisorption, plus particulièrement au dernier stade de la désorption.

Tous ces transferts d'énergie du gaz vers le solide entraînent un échauffement du métal que nous avons visualisé. Cet échauffement est lié à une mauvaise relaxation de l'énergie du catalyseur par convexion en raison de la basse pression de notre réacteur.

7. Conclusion. - Au cours de ce travail, nous avons étudié le processus de catalyse pour une réaction de décomposition et nous avons mis en évidence expérimentalement l'augmentation du taux de décomposition provoqué par l'introduction d'une plaque métallique dans le plasma.

Il s'agit d'un mécanisme de catalyse basé sur l'activation par chimisorption sur le catalyseur des états de faible énergie de $\mathrm{NH}_{3}$.

Ainsi dans le réacteur catalytique, la décomposition de $\mathrm{NH}_{3}$, donc la formation de l'azote, se fait par 2 voies parallèles :
- Une voie homogène en phase gazeuse à partir des états électroniques excités de $\mathrm{NH}_{3}$, contrôlée par l'étape endothermique de dissociation de ces états et favorisée par une température de vibration élevée (pression faible).

- Une voie hétérogène par chimisorption sur la surface du catalyseur, des molécules de faible énergie (possédant une excitation vibrationnelle), beaucoup plus élevée en nombre, cette voie est favorisée par une température de vibration élevée (pression faible).

Les études effectuées sur la fixation de l'azote $[1,2]$ et celle-ci portant sur la formation de l'azote, permettent donc de dégager un phénomène de catalyse commun, à savoir un processus de chimisorption à partir des états de faible énergie qui ne réagissent pas en phase gazeuse.

Nous avons établi l'existence d'une couche limite, au voisinage du catalyseur, présentant une répartition énergétique différente de celle du plasma. Cette couche limite, qui représente la première confirmation expérimentale des calculs théoriques effectués par Wolken [7, 8] rend compte des relations étroites qui existent entre le phénomène catalytique et la nature hors d'équilibre du plasma.

L'étude de l'aspect énergétique du phénomène catalytique, permet de dégager un mécanisme d'activation rétroactif : les molécules d'azote désorbées cèdent par transfert vibration-vibration une partie de leur excitation aux molécules de $\mathrm{NH}_{3}$ qui vont s'adsorber sur la surface. Outre ces transferts, notre étude a montré l'existence de transferts d'énergie du gaz vers le solide et l'influence des propriétés intrinsèques du catalyseur sur ce transfert.

Tous ces transferts d'énergie du gaz vers le solide entraînent un échauffement du métal, que nous avons visualisé et dont l'étude systématique est en cours.

\section{Bibliographie}

[1] Rapakoulias, D., Thèse Université P.-et-M.-Curie (1979).

[2] Amouroux, J., Cavadias, S., Rapakoulias, D., Revue Phys. Appl. 14 (1979) 969.

[3] Rapakoulias, D., Domange, M., Amouroux, J., Revue Phys. Appl. 16 (1981) à paraître (juin 81).

[4] Rapakoulias, D., Amouroux, J., Revue Phys. Appl. 15 (1980) 1251-1259.

[5] Rapakoulias, D., Cavadias, S., Amouroux, J., Revue Phys. Appl. 15 (1980) 1261-1265.

[6] Botchway, G., Venugopalan, M., Phys. Chimie (Wiesbaden) 120 (1980) 103.

[7] McCreery, J., Wolken, G., J. Chem. Phys. 65 (1976) 1310.
[8] McCreery, J., Wolken, G., J. Chem. Phys. 67 (1977) 2551.

[9] Halpern, B., Rosner, D., J. Chem. Soc. Faraday Trans. 74 (1978) 1883.

[10] Wolken, G., Surf. Sci. 82 (1979) 245-254.

[11] Brooks, B. W., Seaport, R. M., J. Appl. Chem. Biotech. 24 (1974) 621.

[12] Eremin, E. N., Rubatsova, V., Russ. J. Phys. Chem. 74 (1973) 356.

[13] Baronnet, J. M., Thèse, Université de Limoges, 1978.

[14] Moeubus, P., J. Electroch. Soc. 122 (1975) 298.

[15] Revoll, M. F., Thèse, Université Paris-VI, Paris, 1981.

[16] Lecuiller, M., Thèse, Université Paris-Sud, 1980.

[17] Polanyi, J. C., Acc. Chem Rech. 5 (1972) 161. 